\title{
FRACTIONAL POWERS OF MOMENTUM OF A SPECTRAL DISTRIBUTION
}

\author{
M. JAZAR
}

(Communicated by Palle E. T. Jorgensen)

\begin{abstract}
In this paper we construct fractional and imaginary powers for the positive momentum $B$ of a spectral distribution and prove the basic properties.

The main result is that for any $\alpha>0,-B^{\alpha}$ generates a bounded strongly continuous holomorphic semigroup of angle $\frac{\pi}{2}$. In particular for $\alpha=1$, using Stone's generalized theorem, if $i B$ generates a $k$-times integrated group of type $O\left(|t|^{k}\right)$ with $\sigma(B) \subset[0,+\infty[$, then $-B$ generates a strongly continuous holomorphic semigroup of angle $\frac{\pi}{2}$. A similar corollary is given in the regularized group situation.
\end{abstract}

\section{INTRODUCTION}

A spectral distribution is a $\check{\mathscr{T}}$-functional calculus of the momentum $B$ (see below), and in [5] we have shown how to define $f(B)$ for any function $f \in$ $\mathscr{C}^{\infty}(V)$, where $V$ is a neighborhood of $\sigma(B)$. This allowed us to define the $\alpha$ th power of $(B+\varepsilon) \quad(\alpha \in \mathbb{C}, \sigma(B) \subset[0,+\infty[$, and $\varepsilon>0)$ as the image of the function $t \mapsto(t+\varepsilon)^{\alpha}$ and finally $B^{\alpha} \equiv \lim _{\varepsilon \rightarrow 0}(B+\varepsilon)^{\alpha}$.

In [5] the authors introduced spectral distributions and gave a generalization of Stone's theorem to a Banach space which connects the momentum of a spectral distribution (see [5] and [16]) to the generator of a temperate integrated group (see [1]) and to the generator of a smooth distribution group (see [3] and [4]). An extensive number of applications of this theorem to the fundamental Cauchy problem in $L^{p}\left(\mathbb{R}^{n}\right)$ is given in [14] and [16]. The relation between spectral distribution and $C$-regularized group (see [8], [9], [10], and [19]) is given in [13].

The typical example of the momentum of a spectral distribution is the Schrödinger operator $\Delta$ in $L^{p}\left(\mathbb{R}^{n}\right), p \in \mathbb{R}$ (see [14]). Comparing with the fact that $\Delta$ is itself the generator of a bounded holomorphic semigroup of angle $\frac{\pi}{2}$, once we have constructed the $\alpha$ th power of $B$, surprisingly we found that if $B$ is the momentum of a spectral distribution, then $-B^{\alpha}$ generates a holomorphic semigroup of angle $\frac{\pi}{2}$. Hence we claim that the originality of this paper lies not only on the new construction of the fractional power which coincides with the standard definition of fractional power of the generator of a holomorphic semigroup (see Remark 3.13) but on the fact that we retrieve the strong continuity

Received by the editors May 19, 1993 and, in revised form, September 30, 1993.

1991 Mathematics Subject Classification. Primary 47B40, 47A60. 
and the analyticity of the semigroup generated by $-B^{\alpha}$ when $i B$ generates a $k$-times integrated group of type $O\left(|t|^{k}\right)$ with $\sigma(B) \subset[0,+\infty[$. This can be considered in some sense as a converse of the result of [12].

Our tools permit us to get the basic properties of $B^{\alpha}$ (like $D\left(B^{\alpha}\right) \subset D\left(B^{\beta}\right)$ for $\operatorname{Re} \alpha \leq \operatorname{Re} \beta$ and $B^{\alpha} B^{\beta} x=B^{\alpha+\beta} x$ ) even if $0 \in \sigma(B)$ (compare with [18]) and give a simple representation of the holomorphic semigroup

$$
G_{\alpha}(z) \equiv \mathscr{E}\left(t \mapsto e^{-z|t|^{\alpha}}\right)
$$

generated by $B^{\alpha}$ (compare with [7] or [20]).

In $\S 4$, using the relation of the momentum of a spectral distribution with regularized group, we give the fractional derivatives formula of $(i B)^{\alpha}$ (see [17]).

\section{SPECTRAL DISTRIBUTION AND ITS BASIC PROPERTIES}

Let $X$ be a Banach space and $\mathscr{L}(X)$ the algebra of bounded linear operators with the uniform operator topology. All the definitions and results of this section can be found in [5].

Definition 2.1. By a spectral distribution we mean a linear continuous mapping $\mathscr{E}$ from $\mathscr{D} \equiv \mathscr{C}_{0}^{\infty}(\mathbb{R}, \mathbb{C})$ into $\mathscr{L}(X)$ which satisfies:

(i) $\mathscr{E}(\varphi \cdot \psi)=\mathscr{E}(\varphi) \mathscr{E}(\psi)$ for all $\varphi, \psi \in \mathscr{D}(\mathbb{R})$.

(ii) For any function $\varphi \in \mathscr{D}$ such that $\varphi(0)=1, \mathscr{E}\left(\varphi_{n}\right)$ converges strongly to the identity $I$, where $\varphi_{n}(t)=\varphi(t / n)$.

Definition 2.2. For any $f \in \mathscr{C}^{\infty}(\mathbb{R})$ let us define $\mathscr{E}(f)$ as an unbounded linear operator by:

$$
D(\mathscr{E}(f)) \equiv\left\{x \in X ; \lim _{n \rightarrow \infty} \mathscr{E}\left(f \phi_{n}\right) x \text { exists for any } \phi \in \mathscr{D} \text { with } \phi(0)=1\right\}
$$

Then for $x \in D(\mathscr{E}(f))$,

$$
\mathscr{E}(f) x \equiv \lim _{n \rightarrow \infty} \mathscr{E}\left(f \phi_{n}\right) x
$$

One can show that this definition is independent of the choice of $\phi$.

Definition 2.3. We say that an unbounded linear operator $B$ admits the spectral distribution $\mathscr{E}$, or $B$ is the momentum of $\mathscr{E}$, if there is a spectral distribution $\mathscr{E}$ such that $B=\mathscr{E}(t)$. Here $t$ denotes the identity function in $\mathbb{R}$.

For $l \in \mathbb{N}$, let $\mathscr{T}_{l}$ denote the completion of $\mathscr{D}$ for the following norm $p_{l}$

$$
p_{l}(\varphi)=\sum_{k \leq l}\left\|t^{k} \frac{d^{k}}{d t^{k}}\right\|_{L^{1}}
$$

and by $\mathscr{T}_{l}=\mathscr{F}^{-1} \mathscr{T}_{l}=\left\{f ; \mathscr{F} f \in \mathscr{T}_{l}\right\}$ equipped with the norm $\Pi_{l}(f)=$ $p_{l}(\mathscr{F} f)$, where $[\mathscr{F} f](t)=\int_{\mathbf{R}} e^{-2 i \pi t s} f(s) d s$. We say that a spectral distribution $\mathscr{E}$ is of degree $l$, if $\mathscr{E}$ can be extended to a linear continuous mapping from $\mathscr{T}_{1}$ into $\mathscr{L}(X)$.

Finally let $\mathscr{T}$ denote the completion of the Schwartz space $\mathscr{S}$ equipped with the system of semi-norms $\left(p_{k}\right)$ and $\mathscr{\mathscr { T }}$ the completion of $\{\varphi \in \mathscr{S} \mid \mathscr{F} \varphi \in \mathscr{T}\}$ in the space of temperate distributions $\mathscr{S}^{\prime}$.

By the inequality $[5,(3.1)]$, for any $k \leq l$ we have $\mathscr{T} \hookrightarrow \mathscr{T}_{l} \hookrightarrow \mathscr{T}_{k}$. 
Remark 2.4 (see [5]). Let $\mathscr{E}$ be a spectral distribution. Then:

(i) For any $x \in D(\mathscr{E}(f))$ we define $f(B) x \equiv \mathscr{E}(f) x$.

(ii) $\mathscr{R} \equiv \bigcup_{\varphi \in \mathscr{D}} \mathscr{E}(\varphi) \subseteq D(\mathscr{E}(f))$ and it is dense in $X$.

(iii) $\mathscr{E}(f)$ is a densely defined closed linear operator.

(iv) If $f=g$ on $\operatorname{sp}(B)$ (spectrum of $B$ ), then $\mathscr{E}(f)=\mathscr{E}(g)$. Thus, we can define $\mathscr{E}(f)$ for any $f \in \mathscr{C}^{\infty}(V)$ where $V$ is a neighborhood of $\operatorname{sp}(B)$.

Lemma 2.5. Let $B$ be the momentum of a spectral distribution $\mathscr{E}$, and let $f$ and $g$ be two functions in $\mathscr{C}^{\infty}(\mathbb{R})$ or $\mathscr{T}$. Then $f(B) g(B) \subset(f \cdot g)(B)$ with $D(f(B) g(B))=D((f g)(B)) \cap D(g(B))$.

Theorem 2.6 (Stone's generalized theorem, see [5]). Let $A$ be a linear densely defined operator on a Banach space $X$. The following are equivalent.

(i) A generates a smooth distribution group of order $k$.

(ii) A generates a $k$-times integrated temperate group $\{G(t)\}_{t \in \mathbb{R}}$ such that $\|G(t)\| \leq C|t|^{k}$ for any $t \in \mathbb{R}$.

(iii) $i A$ admits a spectral distribution of degree $k$.

\section{FRACTIONAL AND IMAGINARY POWERS}

In this section we will show how to construct fractional and imaginary powers of the positive momentum $B$ of a spectral distribution $\mathscr{E}$ of a finite degree $k$. For this we will proceed as in [18]: If $B$ is positive $(\sigma(B) \subseteq[0,+\infty[), \varepsilon>0$, and $(B+\varepsilon)$ is strictly positive, we define $(B+\varepsilon)^{\alpha}$, and then $B^{\alpha}$, as the limit of $(B+\varepsilon)^{\alpha}$ as $\varepsilon$ goes to zero.

In the sequel we denote by $Y(t)$ the Heaviside function: $Y(t)=1$, for $t \geq 0$, and zero elsewhere.

Basic properties of $B^{\alpha}$.

Definition 3.1. The momentum $B$ of a spectral distribution $\mathscr{E}$ is called positive (respectively, strictly positive) if the spectrum of $B$ is contained in $[0,+\infty[$ (respectively, ]0, $+\infty[$ ).

Remark 3.2. Since the spectrum of $B$ is always closed, if $B$ is strictly positive there exists $\varepsilon>0$ such that $\sigma(B) \subseteq[\varepsilon,+\infty[$.

The problem in the positive case is that the function $t \mapsto t^{\alpha}$ is not $\mathscr{C}^{\infty}$ on a neighborhood of $\sigma(B)$.

By Remark 2.4(iv), for any $\alpha \in \mathbb{C}$, we can define the $\alpha$ th power of $(B+\varepsilon)$ :

Definition 3.3. Let $B$ be the positive momentum of a spectral distribution $\mathscr{E}$. For any $\alpha \in \mathbb{C}, \varepsilon>0$, we denote by $(B+\varepsilon)^{\alpha}$ the operator $\mathscr{E}\left(t \mapsto(t+\varepsilon)^{\alpha}\right)$.

Lemma 3.4. Let $B$ be the positive momentum of a spectral distribution $\mathscr{E}, \lambda \in \mathbb{C}$ such that $\operatorname{Im} \lambda \neq 0$. Then:

(1) For all $\varepsilon \geq 0, \mathscr{E}\left(t \mapsto(t+\varepsilon)^{j}\right)=(B+\varepsilon)^{j}, j \in \mathbb{Z}$. In particular, for $\varepsilon>0, \mathscr{E}\left((t+\varepsilon)^{-j}\right) \in \mathscr{L}(X)$ for any $j \in \mathbb{N}$.

(2) For any $\alpha \in \mathbb{C}, \operatorname{Re} \alpha>0$, the function $t \mapsto(\lambda-t)^{-\alpha}$ is in $\check{\mathscr{T}}$.

(3) For any $\alpha \in \mathbb{C}, \varepsilon>0$, the operators $\mathscr{E}\left(t \mapsto(t+\varepsilon)^{\alpha}(\lambda-t)^{-\alpha}\right)$ and $\mathscr{E}\left(t \mapsto(\lambda-t)^{\alpha}(\varepsilon+t)^{-\alpha}\right)$ are bounded. 
(4) For any $\alpha \in \mathbb{C}, \varepsilon>0, D\left((B+\varepsilon)^{\alpha}\right)$ is independent of $\varepsilon$ and

$$
D\left((B+\varepsilon)^{\alpha}\right)=D\left((\lambda-B)^{\alpha}\right)=\operatorname{Im}\left(\mathscr{E}\left((\lambda-t)^{-\alpha}\right)\right) .
$$

(5) For all $\varepsilon>0,(B+\varepsilon)^{-\alpha}$ is bounded for any $\alpha \in \mathbb{C}$ with $\operatorname{Re} \alpha>0$.

Proof. (1) The equality $\mathscr{E}\left((t+\varepsilon)^{j}\right)=(B+\varepsilon)^{j}$ is obvious for $j \in \mathbb{N}$. Now take $j \in \mathbb{N}$ and let us show that $\mathscr{E}\left((t+\varepsilon)^{-j}\right)=(B+\varepsilon)^{-j}$. In fact by Lemma 2.5, $\mathscr{E}\left((t+\varepsilon)^{-j}\right)$ is the inverse of $(B+\varepsilon)^{j}$, and $D\left(\mathscr{E}\left((t+\varepsilon)^{-j}\right)\right)=\operatorname{Im}\left((B+\varepsilon)^{j}\right)=$ $D\left((B+\varepsilon)^{-j}\right)=X$, because $-\varepsilon \notin \sigma(B)$.

(2) See [5, Lemma 4.10.3].

(3) Take $\mu=\lambda+\varepsilon, \mu \in \mathbb{C}$, with $\operatorname{Im} \mu \neq 0$. Let $u=t+\varepsilon$; then by the Taylor formula we have

$$
\begin{aligned}
& (t+\varepsilon)^{\alpha}(\mu-(t+\varepsilon))^{-\alpha}=\left(-1+(1-u / \mu)^{-1}\right)^{\alpha} \\
& =(-1)^{\alpha}+\sum_{j=1}^{n} C_{j, \alpha}(1-u / \mu)^{-j}+O\left(|1-u / \mu|^{-n-1}\right)=c+h(t),
\end{aligned}
$$

where

$$
h(t)=\sum_{j=1}^{n} C_{j, \alpha}(1-u / \mu)^{-j}+O\left(|1-u / \mu|^{-n-1}\right),
$$

$n$ is an integer greater than $\operatorname{Re} \alpha$, and $c$ and $C_{j, \alpha}$ are scalar. Since by the last point the function $t \mapsto(1-(t+\varepsilon) / \mu)^{-j}$ is in $\mathscr{\mathscr { T }}$, the function $h$ is in $\check{\mathscr{T}}$ and $\mathscr{E}\left((t+\varepsilon)^{\alpha}(\mu-(t+\varepsilon))^{-\alpha}\right)=c I+\mathscr{E}(h)$ is bounded. In the same way

$$
\begin{aligned}
(\lambda-t)^{\alpha}(t+\varepsilon)^{-\alpha} & =c^{\prime}+\sum_{j=0}^{n} C_{j, \alpha}^{\prime}((t+\varepsilon) / \mu)^{-j}+O\left(|(t+\varepsilon) / \mu|^{-n-1}\right) \\
& =c^{\prime}+k(t) .
\end{aligned}
$$

Since by the first point $\mathscr{E}\left((t+\varepsilon)^{-j}\right)$ is bounded, $\mathscr{E}\left((t+\varepsilon)^{\alpha}(\mu-(t+\varepsilon))^{-\alpha}\right)$ is bounded too.

(4) By Lemma 2.5 and the equality

$$
(t+\varepsilon)^{\alpha}=(c+h(t))(\lambda-t)^{\alpha},
$$

if $x \in D\left((\lambda-B)^{\alpha}\right)$, then $x \in D\left((B+\varepsilon)^{\alpha}\right)$ (because $(\lambda-B)^{\alpha} x \in D(c I+h(B))$ which is $X)$. Conversely, by the equality

$$
(\lambda-t)^{\alpha}=\left(c^{\prime}+k(t)\right)(t+\varepsilon)^{\alpha},
$$

if $x \in D\left((B+\varepsilon)^{\alpha}\right)$, then $x \in D\left((\lambda-B)^{\alpha}\right)$ (because $(B+\varepsilon)^{\alpha} x \in D\left(c^{\prime}+k(B)\right)$ which is $X)$. So $D\left((B+\varepsilon)^{\alpha}\right)=D\left((\lambda-B)^{\alpha}\right)$ (hence independent of $\varepsilon$ ), and by Lemma $2.5 D\left((\lambda-B)^{\alpha}\right)=\operatorname{Im}\left(\mathscr{E}\left((\lambda-t)^{-\alpha}\right)\right)$.

(5) By this last point and Lemma 2.5 we have

$$
D\left((B+\varepsilon)^{-\alpha}\right)=D\left((\lambda-B)^{-\alpha}\right)=X .
$$

The following theorem gives us the basic properties of the fractional powers of $(B+\varepsilon)$ :

Theorem 3.5. Let $B$ be the positive momentum of a spectral distribution $\mathscr{E}$. Then:

(i) $D\left((B+\varepsilon)^{\beta}\right) \subseteq D\left((B+\varepsilon)^{\alpha}\right)$, for $\alpha, \beta \in \mathbb{C}, \operatorname{Re} \alpha \leq \operatorname{Re} \beta$. 
(ii) $(B+\varepsilon)^{\alpha}(B+\varepsilon)^{\beta} x=(B+\varepsilon)^{\beta}(B+\varepsilon)^{\alpha} x=(B+\varepsilon)^{\alpha+\beta} x$, for $\alpha, \beta \in \mathbb{C}, x \in$ $D\left((B+\varepsilon)^{\gamma}\right)$ where $\gamma \in\{\alpha, \beta, \alpha+\beta\}$ satisfying

$$
\operatorname{Re} \gamma=\operatorname{Max}(\operatorname{Re} \alpha, \operatorname{Re} \beta, \operatorname{Re}(\alpha+\beta)) \text {. }
$$

Proof. (i) Note that if $\operatorname{Re} \alpha \leq 0$, and by the point (5) of the last lemma, the inclusion is obvious. Suppose then $0 \leq \operatorname{Re} \alpha \leq \operatorname{Re} \beta$, and define for $\gamma>0$,

$$
R_{\gamma} \equiv \mathscr{C}\left((\lambda-t)^{-\alpha}\right) \text {. }
$$

By the last lemma, $R_{\gamma} \in \mathscr{L}(X)$, and by the point (4) $D\left((B+\varepsilon)^{\gamma}\right)=\operatorname{Im} R_{\gamma}$. Writing $R_{\beta}=R_{\alpha} R_{\beta-\alpha}$ we conclude.

(ii) Take $\gamma=\{\alpha, \beta, \alpha+\beta\}$, verifying $\operatorname{Re} \gamma=\operatorname{Max}(\operatorname{Re} \alpha, \operatorname{Re} \beta, \operatorname{Re}(\alpha+\beta))$, and $x \in D\left((B+\varepsilon)^{\gamma}\right)$. By (i), $x \in D\left((B+\varepsilon)^{\alpha}\right) \cap D\left((B+\varepsilon)^{\beta}\right) \cap D\left((B+\varepsilon)^{\alpha+\beta}\right)$. Applying Lemma 2.5 and the spectrality of $\mathscr{E}$ we conclude.

Definition 3.6. Let $B$ be the positive momentum of a spectral distribution $\mathscr{E}$. For any $\alpha \in \mathbb{C}$, define $B^{\alpha}$ by

$$
D\left(B^{\alpha}\right) \equiv\left\{x \in D\left((B+\varepsilon)^{\alpha}\right), \text { for } \varepsilon>0 \text {, such that } \lim _{\varepsilon \rightarrow 0}(B+\varepsilon)^{\alpha} x \text { exist }\right\} \text {, }
$$

and for $x \in D\left(B^{\alpha}\right)$,

$$
B^{\alpha} x \equiv \lim _{\varepsilon \rightarrow 0}(B+\varepsilon)^{\alpha} x .
$$

This definition is consistent, since by (4) of Lemma $3.4 D\left((B+\varepsilon)^{\alpha}\right)$ is independent of $\varepsilon$. Moreover the following theorem shows that $D\left(B^{\alpha}\right)=$ $D\left((B+\varepsilon)^{\alpha}\right)$, for any $\varepsilon>0$.

Theorem 3.7. Let $B$ be the positive momentum of a spectral distribution $\mathscr{E}$. For any $\alpha \in \mathbb{C}$ and any $\varepsilon>0$, we have

$$
D\left(B^{\alpha}\right)=D\left((B+\varepsilon)^{\alpha}\right)=D\left((\lambda-B)^{\alpha}\right),
$$

where $\lambda$ is a complex number such that $\operatorname{Im} \lambda \neq 0$.

Proof. By the proof of Lemma 3.4 we have $(t+\varepsilon)^{\alpha}=\left(c+h_{\varepsilon}(t)\right)(\lambda-t)^{\alpha}$ where

$$
h_{\varepsilon}(t)=\sum_{j=0}^{n} C_{j, \alpha}(1-(t+\varepsilon) / \mu)^{-j}+O\left(|1-(t+\varepsilon) / \mu|^{-n-1}\right),
$$

$\lambda \in \mathbb{C}, \operatorname{Im} \lambda \neq 0$, and $\mu=\lambda-\varepsilon$.

Note that, since for any $j \geq 1$ and the function $t \mapsto(1-(t+\varepsilon) / \mu)^{-j}$ goes in $\check{g}$ to the function $t \mapsto(1-t / \lambda)^{-j}$, when $\varepsilon$ goes to zero, then $h_{\varepsilon}$ goes in $\mathscr{\mathscr { T }}$ to a function $h$, when $\varepsilon$ goes to zero. Hence by Lemma 3.4, for $x \in D\left((B+\varepsilon)^{\alpha}\right)=D\left(\mathscr{E}\left((\lambda-t)^{\alpha}\right)\right)$ we have

$$
\begin{aligned}
\lim _{\varepsilon \rightarrow 0}(B+\varepsilon)^{\alpha} x & =\lim _{\varepsilon \rightarrow 0}\left(-I+\mathscr{E}\left(h_{\varepsilon}\right)\right)(\lambda-B)^{\alpha} x \\
& =(-I+\mathscr{E}(h))(\lambda-B)^{\alpha} x,
\end{aligned}
$$

which shows that this limit exists, and thus $D\left(B^{\alpha}\right)=D\left((B+\varepsilon)^{\alpha}\right)$.

Theorem 3.8. Let $B$ be the positive momentum of a spectral distribution $\mathscr{E}$. Then:

(i) $D\left(B^{\beta}\right) \subseteq D\left(B^{\alpha}\right)$, for any complex $\alpha, \beta$, with $\operatorname{Re} \alpha \leq \operatorname{Re} \beta$.

(ii) $B^{\alpha} B^{\beta} x=B^{\beta} B^{\alpha} x=B^{\alpha+\beta} x$, for $\alpha, \beta \in \mathbb{C}, x \in D\left(B^{\gamma}\right)$ where $\gamma \in$ $\{\alpha, \beta, \alpha+\beta\}$ verifying $\operatorname{Re} \gamma=\operatorname{Max}(\operatorname{Re} \alpha, \operatorname{Re} \beta, \operatorname{Re}(\alpha+\beta))$. 
Proof. The first point is obvious applying the preceding theorem and Theorem 3.5(i).

(ii) Take $\gamma \in\{\alpha, \beta, \alpha+\beta\}$ such that $\operatorname{Re} \gamma=\operatorname{Max}(\operatorname{Re} \alpha, \operatorname{Re} \beta, \operatorname{Re}(\alpha+\beta))$, and $x \in D\left(B^{\gamma}\right)$. By (i), $x \in D\left(B^{\alpha}\right) \cap D\left(B^{\beta}\right) \cap D\left(B^{\alpha+\beta}\right)$. By Theorem 3.5, for any $\varepsilon>0$, we have $(B+\varepsilon)^{\alpha}(B+\varepsilon)^{\beta} x=(B+\varepsilon)^{\alpha+\beta} x$. On the other hand, by continuity of $\mathscr{E}$ we have

$$
\begin{aligned}
B^{\alpha} B^{\beta} x & =\lim _{\varepsilon \rightarrow 0}(B+\varepsilon)^{\alpha} \lim _{\tau \rightarrow 0}(B+\tau)^{\beta} x \\
& =\lim _{\varepsilon \rightarrow 0} \lim _{\tau \rightarrow 0}(B+\varepsilon)^{\alpha}(B+\tau)^{\beta} x,
\end{aligned}
$$

so the limit of $(B+\varepsilon)^{\alpha}(B+\tau)^{\beta} x$ exist when $\varepsilon$ and $\tau$ go to zero independently, and is $B^{\alpha} B^{\beta} x$. In particular the limit of $(B+\varepsilon)^{\alpha}(B+\varepsilon)^{\beta} x$ exists when $\varepsilon$ goes to zero and is $B^{\alpha} B^{\beta} x$.

In the following theorem we give the basic fact on the nature of $B^{\alpha}$ :

Theorem 3.9. Let $B$ be the positive momentum of a spectral distribution $\mathscr{E}$ of degree $k$. Then for any $\alpha>0,-B^{\alpha}$ is the generator of a bounded strongly continuous holomorphic semigroup.

Proof. In fact, it is sufficient to show that $-B$ generates a bounded strongly continuous holomorphic semigroup of angle $\frac{\pi}{2}$ (see [11]). To show this, it is sufficient to show that $-e^{i \phi} B$ generates a bounded strongly continuous semigroup, whenever $|\phi|<\frac{\pi}{2}$.

So fix $\phi$ so that $|\phi|<\frac{\pi}{2}$. Define $g(t) \equiv e^{-i \phi|t|}$, and for $s \geq 0, g_{s}(t) \equiv$ $g(s t)$. In order to show that $g$ is in $\check{\mathscr{T}_{k}}$, we write $g(t)=f(t)+f(-t)$, where $f(t) \equiv Y(t) e^{-e^{i \phi} t}$. Using the fact that $\mathscr{F} f(\xi)=\left(i e^{i \phi}+\xi\right)^{-1}$, we get that $\mathscr{F} g(\xi)=2 i e^{i \phi}\left(-e^{2 i \phi}-\xi^{2}\right)$, which is in $\mathscr{T}$. So $g \in \mathscr{\mathscr { T }}$ and for all $s>0$, $\Pi_{k}\left(g_{s}\right)=\Pi_{k}(g)$, where $g_{s}(t) \equiv g(s t)$. Let $G(s) \equiv \mathscr{E}\left(g_{s}\right)$. For $n$ sufficiently large, $\Pi_{k}\left(\left(g_{s}(t)-1\right)(1+1)^{-n}\right)$ converges to zero as $s \rightarrow 0$. Thus $G(s) x \rightarrow x$, for all $x \in \operatorname{Im}\left((1+B)^{-n}\right)=D\left(B^{n}\right)$. Since this is a dense set and $\|G(s)\|$ is uniformly bounded, it follows that $G(s)$ converges strongly to the identity. Again for $n$ sufficiently large, $\frac{d}{d s} g_{s}(t)(1+t)^{-n}=-e^{i \phi}|t| g_{s}(t)(1+t)^{-n}$, where the derivative is taken in the norm $\Pi_{k}$. Using the fact that $B$ is strictly positive, this implies that $\frac{d}{d s} G(s) x=-e^{i \phi} B G(s) x$, for all $x \in D\left(B^{n}\right)$, so that, since $D\left(B^{n}\right)$ is a core for $B,\{G(s)\}_{s \geq 0}$ is a bounded strongly continuous semigroup generated by $-e^{i \phi} B$.

Remark 3.10. In the preceding theorem, what is surprising is that we do not have any restriction on $\alpha>0$, and it is clear that $B^{1}=B$. Thus $B$ is also the generator of a bounded strongly continuous holomorphic semigroup of angle $\frac{\pi}{2}$.

Using Stone's generalized theorem we can write the last theorem as follows.

Corollary 3.11. Let $A$ be the generator of a $k$-times integrated group of type $O\left(|t|^{k}\right)$ such that $\left.\sigma(A) \subset\right] 0 ;-i \infty\left[\right.$. Then for any $\alpha>0,-(i A)^{\alpha}$ is the generator of a bounded strongly continuous holomorphic semigroup of angle $\frac{\pi}{2}$.

Corollary 3.12. Let $A$ be the generator of a uniformly bounded group such that $\sigma(A) \subset] 0 ;-i \infty\left[\right.$. Then for any $\alpha>0,-(i A)^{\alpha}$ is the generator of a bounded strongly continuous holomorphic semigroup of angle $\frac{\pi}{2}$. 
Remark 3.13. In view of Theorem 3.9, the strictly positive momentum of a spectral distribution generates a bounded strongly continuous holomorphic semigroup, so it is important to show that our construction of fractional powers coincides with the classical one. Using Pazy's construction $[20,2.6]$ it suffices to show that for $0<\alpha<1$,

$$
\mathscr{E}\left(t \mapsto t^{-\alpha}\right)=\frac{\sin \alpha \pi}{\pi} \int_{0}^{\infty} s^{-\alpha}(s I+B)^{-1} d s,
$$

where $B$ is the strictly positive momentum of a spectral distribution. To show this write $(s I+B)^{-1}=\mathscr{E}\left(t \mapsto(s+t)^{-1}\right)$, we have

$$
\frac{\sin \alpha \pi}{\pi} \int_{0}^{\infty} s^{-\alpha}(s I+B)^{-1} d s=\frac{\sin \alpha \pi}{\pi} \int_{0}^{\infty} s^{-\alpha \mathscr{E}\left(t \mapsto(s+t)^{-1}\right) d s}
$$

because the appropriate Riemann sums converge in the norm $\Pi_{k}$

$$
\begin{aligned}
& =\frac{\sin \alpha \pi}{\pi} \mathscr{E}\left(t \mapsto \int_{0}^{\infty} s^{-\alpha}(s+t)^{-1} d s\right) \\
& =\frac{\sin \alpha \pi}{\pi} \mathscr{E}\left(t \mapsto \frac{\pi}{\sin \alpha \pi} t^{-\alpha}\right) \\
& =\mathscr{E}\left(t \mapsto t^{-\alpha}\right) .
\end{aligned}
$$

Remark 3.14. When an operator $A$ generates a $k$-times integrated semigroup that is $O\left(t^{k}\right)$, then it is not hard to show (see [5, Lemma 4.10(1)] or [15, Remark after Theorem 4.1]) that there exists a constant $M$ so that

$$
\left\|(s+A)^{-n}\right\| \leq \frac{M}{s}, \quad \text { for all } s>0,
$$

so that we may define $A^{\alpha}$ in the usual way (see Pazy [20] and Remark 3.13). When $B$ is the positive momentum of a spectral distribution, as in this section, then by Theorem $2.6 i B$ generates a $k$-times integrated group that is $O\left(|t|^{k}\right)$; thus we could use this construction to define $B^{\alpha} \equiv(-i)^{\alpha}(i B)^{\alpha}$; as in Remark 3.13 , this definition may be shown to be equivalent to the definition of this section. One could then use the known properties of fractional powers of operators satisfying $(*)$ to obtain Theorem 3.8. But it is of interest to see Theorem 3.8 deduced by a functional calculus argument, as is done here. Lemma 3.4 will also be needed for the next section.

\section{Fractional DERIVATIVES}

Take $A$ the generator of a uniformly bounded semigroup $\{G(t)\}_{t \geq 0}$; then in lieu of constructing $A^{\alpha}$ as the generator of the semigroup $\left\{G_{\alpha}(t)\right\}_{t \geq 0}$ one can show that $A^{\alpha}$ could be the $\alpha$ th derivative of $A$, i.e.,

$$
A^{\alpha} x=\lim _{t \rightarrow 0}((G(t)-I) / t)^{\alpha} x
$$

where this limit exists if and only if $x \in D\left(A^{\alpha}\right)$ (see, for example, [17]).

In our case, when $B$ is the momentum of a spectral distribution $\mathscr{E}$ of degree $k$, we will show an analogous formula, using the relation with the $(\lambda-i B)^{-k}$ regularized group (see [13, Theorem 3.1] or [16, théorème IV.1.1]). 
Theorem 4.1. Let $B$ be the strictly positive momentum of a spectral distribution $\mathscr{E}$ of degree $k$. Then for any $\alpha \in \mathbb{C}$ with $\operatorname{Re} \alpha>0,(i B)^{\alpha}$ is the $\alpha$ th derivative of the $(\lambda-i B)^{-k}$-regularized group $\left\{W_{\lambda}(s)\right\}_{s \in \mathbb{R}}$ defined by

$$
W_{\lambda}(s) \equiv \mathscr{E}\left(t \mapsto(\lambda-t)^{-k} \exp (i s t)\right),
$$

i.e.

$$
\begin{array}{r}
D\left(B^{\alpha}\right)=\left\{x \text { such that } \lim _{s \rightarrow 0} \mathscr{E}\left(\left((\lambda-t)^{-k}(\exp (\text { ist })-1) / s\right)^{\alpha}\right) x\right. \text { exists } \\
\text { and is in } \left.D\left(\mathscr{E}\left((\lambda-t)^{\alpha k}\right)\right)\right\},
\end{array}
$$

and for $x \in D\left(B^{\alpha}\right)$,

$$
(i B)^{\alpha} x=\mathscr{E}\left((\lambda-t)^{\alpha k}\right) \lim _{s \rightarrow 0} \mathscr{E}\left(\left((\lambda-t)^{-k}(\exp (i s t)-1) / s\right)^{\alpha}\right) x
$$

where $\lambda \in \mathbb{C} \backslash \mathbb{R}$.

Proof. Since the case $k=0$ is immediate, let us suppose $k>0$. By Lemma 3.4.2 the function $t \mapsto(\lambda-t)^{-\alpha k}$ is in $\check{\mathscr{T}}$, and using Lemma 2.5 the operator $C_{\alpha} \equiv \mathscr{E}\left((\lambda-t)^{-\alpha k}\right)$ is a bounded injective operator. Let us denote by $C_{-\alpha}$ its inverse.

Now take $x \in D\left(B^{\alpha}\right)$, for $s$ positive we have

$$
\begin{aligned}
& \mathscr{E}\left(\left((\lambda-t)^{-k}(\exp (i s t)-1) / s\right)^{\alpha}\right) x-C_{\alpha}(i B)^{\alpha} x \\
& \quad=\mathscr{E}\left((\lambda-t)^{-\alpha k}\left(((\exp (i s t)-1) / s)^{\alpha}-(i t)^{\alpha}\right)\right) x .
\end{aligned}
$$

Since the function of the right-hand member satisfies the hypothesis of $[5$, Lemma 4.5] with

$$
f(s, t) \equiv(\lambda-t)^{-\alpha k}\left(((\exp (i s t)-1) / s)^{\alpha}-(i t)^{\alpha}\right),
$$

this shows the first inclusion.

Conversely, take $x$ such that the limit exists and is in $D\left(\mathscr{E}\left((\lambda-t)^{\alpha k}\right)\right)$, and let $x_{n} \equiv \mathscr{E}\left(\phi_{n}\right) x$, where $\phi_{n}$ are as in Definition 2.1(ii). Since for all $n$, functions $t \mapsto \phi_{n}(t)\left(i t(\lambda-t)^{-k}\right)^{\alpha}$ and $t \mapsto \varphi_{n}(t)\left(\left((\lambda-t)^{-k}(\exp (i s t)-1) / s\right)^{\alpha}\right)$ are in $\check{\mathscr{T}}_{k}$ (see Lemma 3.4), and applying [5, Lemma 4.5] with the function

$$
f(s, t) \equiv \phi_{n}(t)\left(\left((\lambda-t)^{-k}(\exp (i s t)-1) / s\right)^{\alpha}\right)
$$

we get

$$
\lim _{s \rightarrow 0} \mathscr{E}\left(\left((\lambda-t)^{-k}(\exp (i s t)-1) / s\right)^{\alpha}\right) x_{n}=\mathscr{E}\left(\left(i t(\lambda-t)^{-k}\right)^{\alpha}\right) x_{n} .
$$

Now define, for fixed $\alpha$, the family of operators

$$
S(s) \equiv \mathscr{E}\left(\left((\lambda-t)^{-k}(\exp (i s t)-1) / s\right)^{\alpha}\right) \quad(s \geq 0) .
$$

Then we have

$$
\begin{aligned}
\mathscr{E}\left(t^{\alpha} \phi_{n}\right) x-\mathscr{E}\left(t^{\alpha} \phi_{m}\right) x & =(\lambda-B)^{\alpha k}\left[\lim _{s \rightarrow 0} S(s)\left(x_{n}-x_{m}\right)\right] \\
& =\mathscr{E}\left(\phi_{n}-\phi_{m}\right)\left((\lambda-B)^{\alpha k} \lim _{s \rightarrow 0} S(s) x\right),
\end{aligned}
$$

thus $\mathscr{E}\left(t^{\alpha} \phi_{n}\right) x$ is Cauchy and, hence convergent, so that $x \in D\left(B^{\alpha}\right)$. 


\section{ACKNOWLEDGMENT}

The author would like to thank Professor R. deLaubenfels for his several and useful remarks and suggestions concerning this paper.

\section{REFERENCES}

1. W. Arendt and H. Kellermann, Integrated solutions of Volterra integro-differential equations and applications, Semester-bericht Functionalanalysis, Tübingen, Sommersemester, 1987.

2. M. Balabane, Puissances fractionnaires d'un opérateur d'un semi-groupe distribution régulier, Ann. Inst. Fourier (Grenoble) 26 (1976), 157-203.

3. M. Balabane and H. Emamirad, Smooth distribution group and Schrödinger equation in $L^{p}\left(\mathbb{R}^{n}\right)$, J. Math. Anal. Appl. 70 (1979), 61-71.

4. __ Pseudo-differential parabolic systems in $L^{p}\left(\mathbb{R}^{n}\right)$, Contributions to Non-Linear P. D. E., Pitman Res. Notes Math. Ser., vol. 89, Longman Sci. Tech., Harlow, 1983.

5. M. Balabane, H. Emamirad, and M. Jazar, Spectral distributions and generalization of Stone's Theorem to the Banach space, Acta Appl. Math. 31 (1993), 275-295.

6. Balakrishnan, Fractional powers of closed operators, Pacific J. Math. 10 (1960).

7. S. Bochner, Diffusion equations and stochastic processes, Proc. Nat. Acad. Sci. U.S.A. 35 (1949), 369-370.

8. G. Da Prato, Semigruppi regolarizzabili, Richerche Mat. 15 (1966), 233-248.

9. R. deLaubenfels, Integrated semigroups, C-semigroups and the abstract Cauchy problem, Semigroup Forum 41 (1990), 83-95.

10. _ C-semigroups and the abstract Cauchy problem, J. Funct. Anal. 111 (1993), 44-61.

11. _ Totally accretive operators, Proc. Amer. Math. Soc. 103 (1988), 551-556.

12. R. deLaubenfels and K. Boyadzhiev, Boundary values of holomorphic semigroups, Proc. Amer. Math. Soc. 118 (1993), 113-118.

13. R. deLaubenfels, H. Emamirad, and M. Jazar, Regularized scalar operators (submitted).

14. H. Emamirad and M. Jazar, Application of spectral distributions to some Cauchy problems in $L^{p}\left(\mathbb{R}^{n}\right)$, Trends in Semigroup Theory and Evolution Equations (P. Clément, ed.), Marcel Dekker, New York, 1991, pp. 143-151.

15. M. Hieber, Laplace transforms and $\alpha$-times integrated semigroups, Forum Math. 3 (1991), 595-612.

16. M. Jazar, Sur la théorie de la distribution spectrale et applications aux problèmes de Cauchy, Thèse de l'Université de Poitiers, 1991.

17. O. E. Lanford III and D. W. Robinson, Fractional powers of generators of equicontinuous semigroups and fractional derivatives, J. Austral. Math. Soc. Ser. A 46 (1989), 437-504.

18. C. Martinez, M. Sanz, and L. Marco, Fractional powers of operators, J. Math. Soc. Japan (2) 40 (1988).

19. I. Miyadera and N. Tanaka, Some remarks on $C$-semigroups and integrated semigroups, Proc. Japan Acad. Ser. A 63 (1987).

20. A. Pazy, Semigroups of linear operators and applications to partial differential equations, Springer, New York, 1983.

21.' K. Yosida, Functional analysis, Springer-Verlag, Berlin, 1964.

Universite de Poitiers, Département de Mathématiques, 40 Avenue du Recteur, Pineau 86022 Poitiers, France 